\title{
Designing for Technicians Working in the Field:
}

\section{Usability Heuristics for Mobile Application Design}

\author{
Andrew Johnston \\ School of Software \\ University of Technology Sydney \\ andrew.johnston@uts.edu.au
}

\author{
Michelle Pickrell \\ Faculty of Design, Architecture \& Building \\ University of Technology Sydney \\ michelle.pickrell@student.uts.edu.au
}

\begin{abstract}
Mobile applications are frequently used by technicians and logistics personnel to access documentation and communicate and $\log$ information about the work they do in the field. Currently, however, there are no context-specific usability heuristics for use by designers who are building mobile applications for this sector. By conducting contextual inquiries with technicians and logistics personnel who use mobile applications for their day to day work, we identified specific usability issues affecting the use of these applications. From this research, we propose a set of eight heuristics for use by designers and developers creating mobile applications for users in this area.
\end{abstract}

\section{Author Keywords}

Usability heuristics; mobile devices; context of use; field technician.

\section{ACM Classification Keywords}

H5.m. Information interfaces and presentation (e.g., HCI): Miscellaneous.

\section{INTRODUCTION}

This paper examines the user experiences of field technicians with applications running on mobile devices. We use the term 'field technician' broadly to refer to people working primarily in technical roles who use mobile devices extensively to organize and record their activities. In this paper we focus on contextspecific issues that impact upon field technicians' experiences with the applications they use and on their ability to complete their work effectively.

Field technicians work with mobile applications to organise and record their activities in the field. We argue that existing usability heuristics for the design of mobile applications do not consider the specific contextual limitations of technicians working in the field. To explore this area, we conducted a series of contextual inquiries to help understand the strengths and weaknesses of a range of different applications used by different professionals in the field. Users ranged from field technicians - who work to repair and/or install equipment - to delivery personnel.

To ensure that mobile applications for field technicians are designed to best suit their needs, it is important to thoroughly understand the users' context of use, the users' goals and the tasks they are performing on the application (Inostroza et al., 2016). In addition, Suchman has shown that the way applications are used in the real world may not match up with the designers' expectations (Suchman, 1987). It is therefore important to carefully examine their actual use in the field.

The research goals for the study presented in this paper are:

- investigate the effectiveness and efficiency of mobile applications used by field technicians.

- explore specific issues faced by technicians using applications in the field.

- propose new, specific, heuristics for mobile applications used by field technicians based on findings from the above.

This study focuses on smart phones and tablets such as Apple's iPhone and iPad, and Android mobile devices.

\section{USABILITY HEURISTICS}

Nielsen proposed a set of ten heuristics for graphical user interfaces (GUI's) on desktop computers (Nielsen et al., 1994).. Heuristics have two main uses; they can be used during the creation of a product as best practice guidelines and/or used as a tool to validate a design (Salazar et al., 2013).

Heuristic evaluation is an inexpensive and relatively fast method for finding usability problems which can be performed without the need to involve the end users of the product (Gresse von Wangernheim et al, 2016; Bertini et al., 2009). It is estimated that it can detect between 75 and $80 \%$ of usability flaws (Nielsen, 1994; Nielsen et al., 1990). However, heuristic evaluation does not fully consider the specific conditions under which users will actually use the system (Ji et al., 2006).

One of the most important elements of heuristic evaluation is choosing the most relevant set of heuristics for the context (Inostroza et al, 2016). A generic heuristic review can be conducted, however 
these may miss domain specific problems (Inostroza et al, 2016; Gresse von Wangernheim et al, 2016).

\section{Mobile heuristics}

Designing for mobile devices is difficult for a range of reasons including the physical limitations of the devices (Nielsen et al., 2013). These include the small screen size, which often needs to display large amounts of information (Inostroza et al, 2012; Tsiaousis et al., 2010). Alongside this, mobile devices have limited input controls and capabilities which hinder the quality of the interaction (Salazar et al., 2013; Tsiaousis et al., 2010). Slow connections and short battery life are other constraints of mobile devices (Sovio et al., 2007).

There is only a limited set of specific, current usability guidelines for mobile applications. This is mainly due to the fact that the technology has evolved very quickly (Yanez Gomez et al., 2014; Ji et al., 2006). In the past decade mobiles have advanced from being a device to make calls and are now complex, interactive multimedia systems (Ji et al., 2006). Therefore, many of the heuristic guidelines for older mobile devices are no longer relevant. However, Gomez (2014) proposes that we can re-use guidelines from the literature and adapt them, because the purpose behind the heuristic is derived from human behaviour rather than technology (Budiu et al., 2011).

To create heuristics for the design of successful mobile interactions it is important to understand the context in which they take place (Savio et al., 2007). For example, a set of heuristics used to evaluate a news or social media application on a phone may be very different to those used to validate an application used by an elevator technician. At a high level, 'context' includes a range of aspects including location and time of use as well as the environment in which the person is using their device (Bertini et al, 2009; Longoria, 2004). In particular, environmental aspects such as noise levels and lighting conditions should be taken into account (Longoria, 2004)

Considering how these environmental factors affect the user when they are interacting with the mobile application is of great importance. For example, there are many different distractions which can occur during mobile use. Firstly, visual distractions can be caused by a change in light which causes the user to have to reposition (Yi et al, 2005). Mobile users may also be exposed to several auditory distractions, such as people talking to them, during the use of their device (Tsiaousis et al, 2010). Alongside these factors, users may be multitasking, such as talking to someone or completing other tasks (Longoria, 2004). This shows that heuristics must be carefully selected or sourced so that they are relevant to the specific context that is being designed for (Holzinger, 2005).

Thus far, little research exists that proposes heuristics to better evaluate today's smartphone application characteristics and limitations (Gresse von Wangenheim et al., 2016). Salazar completed a systematic literature review which identified that there were few 'new' heuristics for the mobile context and proposed instead a range of slight adaptations on Nielsen's 'traditional' heuristics (Salazar et al., 2013).

\section{MATERIALS AND METHODS}

A qualitative study design was used to get a detailed understanding of the technician's personal perspectives on the technology that they use on a day to day basis. Contextual Inquiries were conducted with eight technicians who work in the field and use applications as a way of communicating and logging the information relevant to them and their company. In all, approximately 25 hours of interviews and observations were conducted.

Contextual interviews were performed within the context of the interviewee's workplace. All interviews were conducted in the field. The researchers travelled with the technicians between jobs to get an understanding of how the different applications are used for different types of jobs. The researchers focused on the impacts of the interviewees' work environment on their use of the applications. Particular attention was paid to factors which impeded interviewees' ability to work effectively and efficiently and/or comply with health and safety requirements.

All participants were male and had been working in their current role between 8 months and 15 years. Some participants conducted their work outdoors or in their cars, while others conducted their work indoors, for example working in lift wells.

\section{FINDINGS}

This section outlines the results from the contextual interviews. Affinity diagramming (Beyer et al., 1998) was used to identify the themes in the data.

\section{Interface}

During the interviews we identified three main issues with the interface design of the applications used by participants. These include poor colour contrast; small button size and unintuitive button placements; and very long forms.

The colours used for some applications made it difficult for the user to see the details of the interface. In some cases this was blue buttons on a black background. This is exacerbated by the glare created by the sunlight on the screen as well as additional glare which resulted from the user wearing a high visibility safety vest. It was observed that technicians would have to move into a shaded area under a tree or in the shadow of their vehicle to increase the visibility of the screen.

Small button size was another factor often seen in the applications used in the field. In many cases mobile touch target sizes (Nielsen et al, 2013) were not incorporated into the design, making it difficult for technicians to see the relevant button they were meant to press. This included important buttons such as the ability to save the form. In addition, the placement of buttons was sometimes unintuitive. For example, the button to submit a form was placed at the top of the 
form so the user was forced to scroll back up after entering data.

It was observed that technicians and delivery workers often had to input a lot of detailed information into the app. This was presented in very different formats. In some cases this was long forms with between 20 and 30 questions. In other cases it was a few open text fields where technicians were able to openly add whatever information they thought was necessary. Technicians commented that it was quite time consuming writing the information through the application on the phone due to the small screen and keyboard. In some cases the technicians would write notes in an email to send to themselves. They would then access the information later and copy it across to a web version of the same tool using a full size screen and keyboard.

\section{Hardware}

The main hardware issues observed concerned battery life, screen responsiveness and the effects of weather conditions. Alongside these issues, potential improvements were identified in the ways that phones' Global Positioning System (GPS) and camera were used.

Technicians commented that due to the glare on the screen being particularly bad, it was necessary to have the phone's screen brightness at full. This resulted in the battery draining quite quickly. While technicians often had car phone chargers so that they could recharge their device in the car, they were not in the vehicles for long enough in between jobs to fully re-charge their phones. When the phone's battery was low, their camera would become unresponsive. ${ }^{1}$ The lack of camera functionality would then prevent the technicians from completing tasks which required images, such as work site audits.

Other technicians commented that the scanning functionality of their device did not work when it got wet, which happened frequently in rainy weather. For delivery drivers, whose primary work is based on scanning a barcode, the scanning ability is integral to them completing their day-to-day work.

It was also observed that GPS could have been incorporated into the applications to improve efficiency. For example, in some cases the technician had to type an address into the application multiple times where the GPS could be used to pre fill this for the technician based on their current location.

The observations and interviews showed that native applications gave users the ability to upload images far more efficiently than web-based applications. Users were able to take a photograph directly in the app, rather than having to take a photo using the phone's camera app, switch back to the browser, select the upload button, locate the image in the phone's image gallery and then upload it.

1 Some operating systems make the camera unresponsive when charge is low to prolong battery life.

\section{User experience and user control}

In some cases the applications did not give the technicians the control and freedom they needed to do their jobs. Many of the applications had shortfalls around the user experience, and as a result technicians had created work-arounds that allowed them to complete their jobs more easily.

In many cases the technicians needed information when on the job - for example, technical documentation for a particular piece of technology that they were working on. The applications used by these technicians often did not contain this documentation, and so in order to complete their work the technician would have to call their head office to ask office staff to look up certain information in manuals, or try to find the documentation online. In both situations, time is wasted and additional staff resources are required. If documentation cannot be found quickly, jobs which might otherwise be completed successfully on the spot must be deferred to a future visit. Technicians commented that they did not understand why this information is not available through the app. ${ }^{2}$

It was also observed that some technicians had to work across several different applications. This was due to the fact that multiple companies were working together. Each company had their own application and database and therefore technicians often had to input similar information about a particular job in both applications in order to fulfill reporting requirements.

\section{Use of data}

It wasn't always clear to technicians what the information they were recording was being used for. As a result, technicians would not always log enough information into the system or provide the relevant information needed. In some cases, it is very important for technicians to know what work has previously been done on particular pieces of equipment, as this will impact on how they approach a repair task for example. Where this past work has not been recorded in sufficient detail, the time taken for future repairs - and the quality of the work - may be impacted significantly.

Pragmatic information such as how to access a particular worksite was identified as being important, as was information about the client's history with the company and issues that had arisen during past jobs with that client. In other cases the ways that data was used was very obvious to the user. For example, some of the technicians used their applications to $\log$ the number of jobs they had done and this directly related to the financial payment they were given. In some cases there was a strong workplace performance aspect of the

\footnotetext{
2 There may be valid reasons (relating to copyright or the desire to secure an organisation's intellectual property for example) for not making some documentation available in easily copyable formats such as PDF. It may, however, be possible to supply this information embedded in an application without risking piracy.
} 
applications that they use and the data collected was reflected back to the teams on a daily basis.

\section{PROPOSED HEURISTICS}

Below are eight heuristics which are specific to the design of mobile applications for use by technicians working in the field. These heuristics are based on this research, however they have not yet been tested. It is recommended that they be used alongside other methods to validate application design including usability testing.

H1 - Ensure high contrast colour combinations are used for ease of visibility outdoors.

Technicians often had difficulty seeing the content on the mobile screen when outdoors. This was due to glare from both the surrounding environment as well as their high visibility shirts. Colour combinations for outdoor use, such as dark text on a white or cream background can result in easier legibility.

\section{H2 - Design applications to reduce strain on device batteries}

Due to the devices needing to be set with the highest backlight display for visibility, technicians commented that their devices quickly ran out of battery. They often had car chargers but were not in their cars for long enough to charge their devices adequately. Designing applications using display colours, which reduce the need to have backlighting on full, can reduce power consumption.

\section{H3 - Provide users with multiple ways to provide input}

One common barrier to entering information into the application was the small screen and small keyboard of the mobile devices. This often resulted in technicians writing high-level notes in the application and having to return to the system later in the day when they were at their desktop computer to write up their full report. Providing additional ways for this information to be captured, such as such as image, audio recordings or structured text, allows for easier input by technicians.

H4 - Provide technicians with access to activity histories

A single client or worksite is often visited many times by a wide range of different technicians. Some technicians, have a history of numerous past jobs with particular clients. Information learned by a technician about a particular client should be made available to other technicians working with that client in the future via their app/s. The same applies to pieces of equipment which may be serviced or repaired many times during their working life.

H5 - Provide technicians with the technical documentation they need to do their jobs through the app

In some cases technicians did not have easy access to the technical documentation they needed to do their job, such as safety guidelines or equipment manuals. This was often frustrating for technicians, as they would have to search online or call head office to arrange to have it sent. Incorporating this information into an app helps ensure it can easily be accessed.

H6 - Ensure the application is able to locally save any relevant data

In a few cases, delivery personnel lost over an hours worth of information that they had input into their system as it had not been saved on their device. This resulted in the technician having to re-do this work. To reduce the risk of data being lost, ensure that the technology has the capacity to function offline without issue for at least the duration of the work. Data should then be synced automatically when an internet connection becomes available.

H7 - Provide technicians with an understanding of what the data they input will be used for

Technicians commented that they were not always sure what the information they were inputting into the application would be used for. This resulted in some technicians not always adding the necessary detail. Technicians also commented that they would appreciate having access to past job information when they returned to a customer. Providing technicians with an outline of what the data they input is used for, and access to this information when it is most useful (see $\mathrm{H} 4$ and H5), helps them to understand what information they are required to input and encourages them to do this fully and completely.

H8 - Design application workflow around the technicians' work practices instead of the technical structures of back-end systems

The structures and limitations of back-end systems are often reflected in the technicians' experiences with their applications. The design of the application workflow should be based on the needs and existing work practices of the technician, rather than the technical structures of existing systems.

\section{CONCLUSION}

This study focused on the use of mobile applications by technicians working in the field. We found that there are no existing usability heuristics designed for this specific context. Contextual inquiries were conducted to better understand this specific context of use. Based on the findings from these inquiries, a set of eight heuristics have been proposed for use by designers and developers working in the area. These proposed context-specific heuristics are intended to be used alongside 'traditional' usability heuristics for interaction design. Using both sets of heuristics, alongside other validation methods, such as usability testing, should result in more usable applications for technicians in the field.

\section{ACKNOWLEDGEMENTS}

We would like to thank all the organisations and field technicians who participated in this study. 


\section{REFERENCES}

Bertini, E., Catarci, T., Dix, A., Gabrielli, S., Kimani, S. and Santucci, G. Appropriating Heuristic Evaluation for Mobile Computing. International Journal of Mobile Human Computer Interaction 1, 1 (2009), 2041.

Beyer, H. and Holtzblatt, K. Contextual design: Defining customer-centered systems. San Francisco, CA: Morgan Kaufmann. (1998).

Budiu, R. and Nielsen, N. Usability of iPad Apps and Websites, Nielsen Norman Group, 2nd Edn, (2011).

Gresse von Wangenheim, C., Witt, T. and Borgatto, A. et al. A Usability Score for Mobile Phone Applications Based on Heuristics. International Journal of Mobile Human Computer Interaction 8, 1 (2016), 23-58.

Holzinger, A. Usability engineering methods for software developers. Communications of the ACM 48, 1 (2005), 71-74.

Inostroza, R., Rusu, C., Roncagliolo, S., Jimenez, C. and Rusu, V. Usability Heuristics for Touchscreenbased Mobile Devices. In Proc. ITNG '12, IEEE, (2012), 662-667.

Inostroza, R., Rusu, C., Roncagliolo, S., Rusu, V. and Collazos, C. Developing SMASH: A set of SMArtphone's uSability Heuristics. Computer Standards \& Interfaces 43, (2016), 40-52.

Ji, Y., Park, J., Lee, C. and Yun, M. A Usability Checklist for the Usability Evaluation of Mobile Phone User Interface. International Journal of Human-Computer Interaction 20, 3 (2006), 207-231.

Longoria, R. Designing software for the mobile context. Springer, New York, (2004).
Nielsen, J., and Molich, R., Heuristic evaluation of user interfaces. In Proc. CHI 1990, ACM Press (1990), 249-256.

Nielsen, J., Heuristic Evaluation, In Nielsen, J. and Mack, R.L., Usability inspection Methods, New York: Wiley (1994), 25-62.

Nielsen, J. and Budiu, R. Mobile usability. New Riders, Berkeley, CA, (2013).

Salazar, L., Lacerda, T., Nunes, J. and Gresse von Wangenheim, C. A Systematic Literature Review on Usability Heuristics for Mobile Phones. International Journal of Mobile Human Computer Interaction 5, 2 (2013), 50-61.

Savio, N. and Braiterman, J. Design Sketch: The Context of Mobile Interaction. In Proc. MobileHCI (2007), 284-286.

Suchman, L. A. 1987, Plans and situated actions: the problem of human-machine communication, Cambridge University Press, New York, NY, USA.

Tsiaousis, A. and Giaglis, G. An Empirical Assessment of Environmental Factors that Influence the Usability of a Mobile Website. In Proc. ICMB-GMR '10. IEEE (2010), 161-167.

Yáñez Gómez, R., Cascado Caballero, D. and Sevillano, J. Heuristic Evaluation on Mobile Interfaces: A New Checklist. The Scientific World Journal 2014, (2014), 1-19.

Yi, J.S., Choi Y.S., and Jacko, J. A. Context awareness via a single device-attached accelerometer during mobile computing. In Proc. MobileHCI '05, ACM Press, (2005), 303-306. 